\title{
DIGITAL SAFETY NETTING TOOLS: FUTURE TECHNOLOGIES TO REDUCE AVOIDABLE DEATHS IN CHILDHOOD
}

Neill, Sarah; Roland, Damian; Thompson, Matthew; Palmer-Hill, Sue; Bayes, Natasha; Mullins, Laura; Turner, Tracy; Tavare, Alison; Lakhanpaul, Monica.

\section{Background}

Children's use of urgent care services continues to increase.

If families are to access the right services at the right time they need access to information to inform their decision making. Providing a safety net of information has the potential to reduce morbidity and avoidable mortality (Jones et al 2013) and has been shown to reduce re-consultation safely (Maguire et al 2011).

Safety netting tools for the future need to be designed with digital devices in mind.

\section{"Our research programme aims} to provide parents with information they can use to help them determine when to seek help for an acutely ill child"

\section{Methods}

Our evidenced programme of intervention co-design included:

A systematic review of existing interventions; qualitative exploration of safety netting information used by parents and professionals; development of the content and delivery modes for the intervention; video capture of children with acute illness; and digital safety netting tool development using consensus methodology.

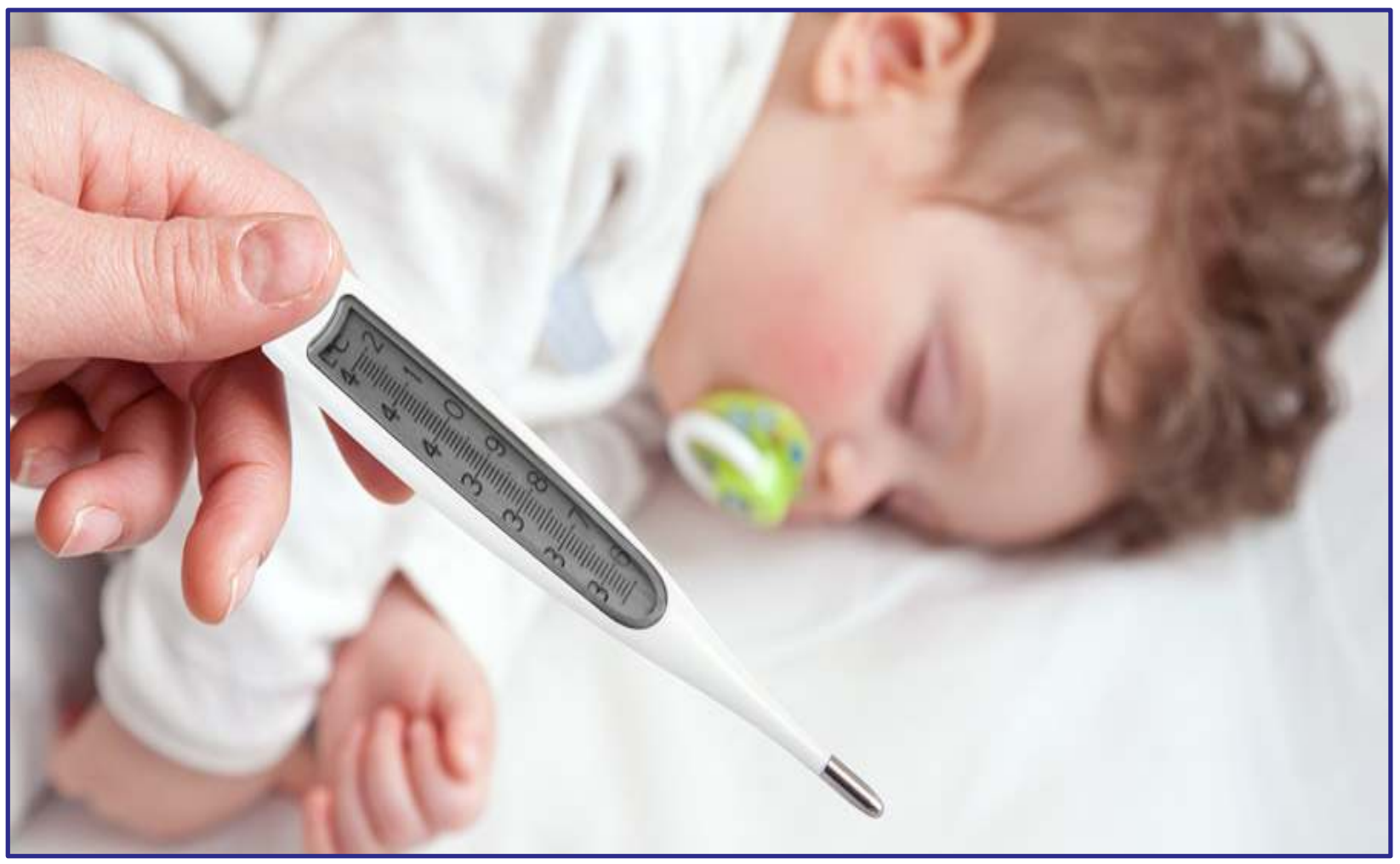

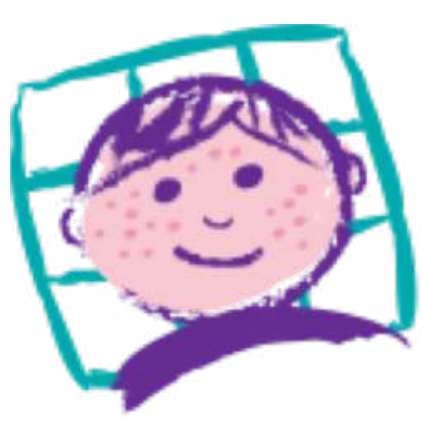

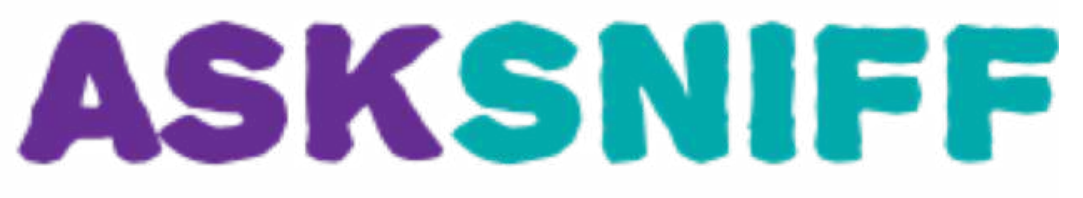

ACUTELY SICK KID SAFETY NETTING INTERVENTIONS FOR FAMILIES

\section{Results}

Our findings demonstrated the need for professionally endorsed and co-produced digital safety netting resources focussing on symptoms of acute childhood illness. Working with parents and professionals we have developed an evidenced co-produced digital safety netting tool supported by video materials, enabling parents to see symptoms for real.

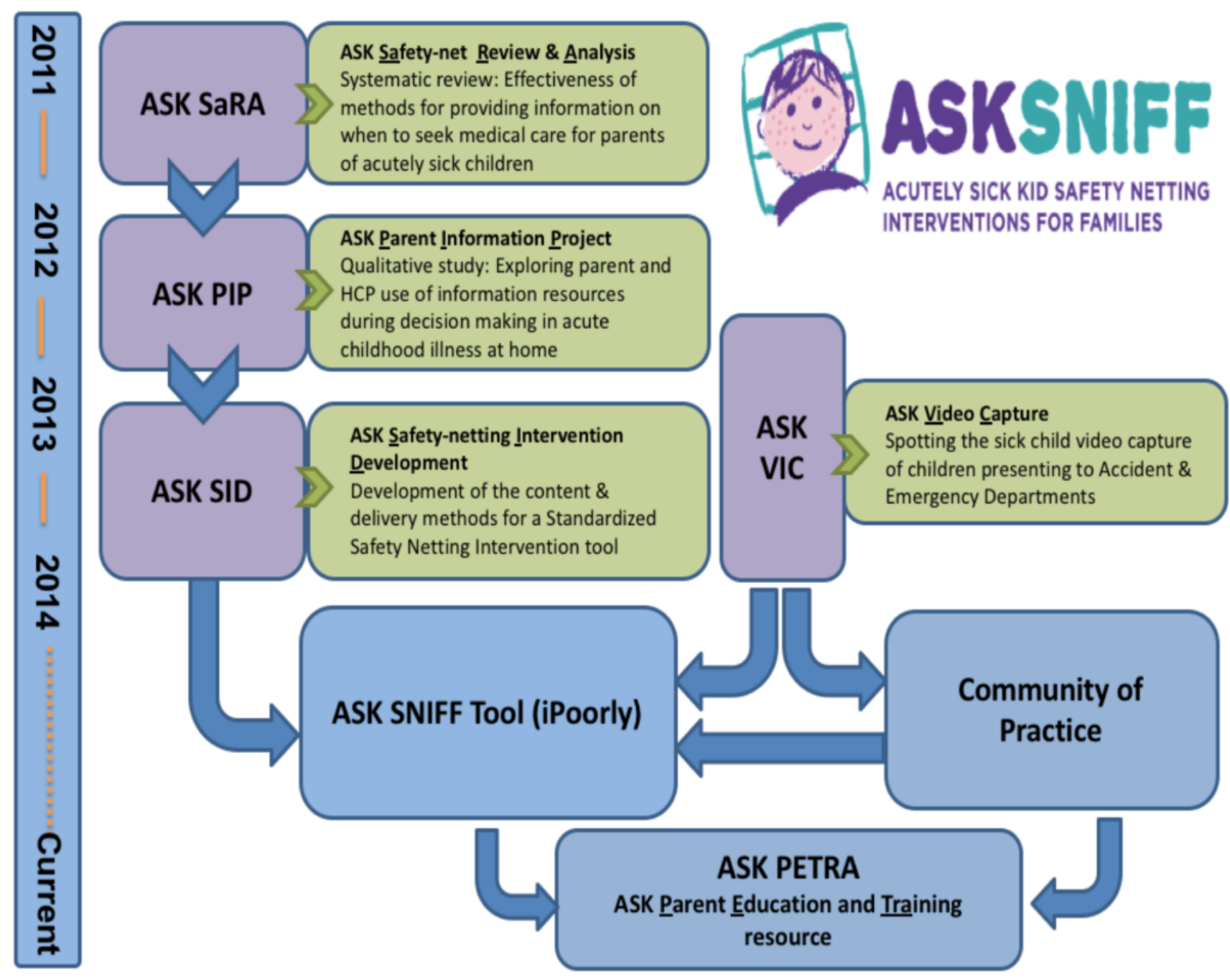

\section{Conclusion}

Digital safety netting tools will provide parents and professionals with instant access to information to support families' access safety netting at home and to recognise symptoms which require urgent or emergency care. All such tools need to be developed with future technologies in mind.

\section{http://asksniff.org.uk}

Dr. Damian Roland

dr98@Leicester.ac.uk

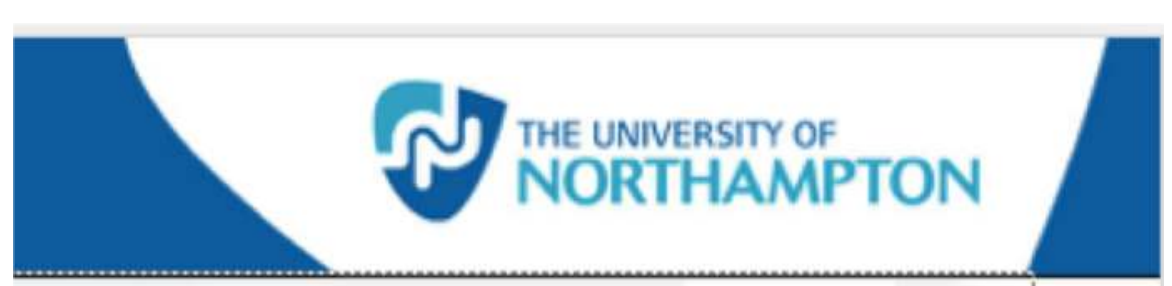

Northamptonshire Healthcare WiS NHS Foundation Trust

M

